Therefore by Lemma 2

$$
\begin{aligned}
m U & >\frac{1}{416} \frac{c^{2}}{4 M^{2}} m G \\
& >\frac{1}{1664} \frac{c^{2}}{M^{2}} m E_{3} .
\end{aligned}
$$

As $U$ is included in $H$ we conclude from (10) that

$$
m\left(E_{2} \times U\right)>0
$$

which is impossible since by the definition of $E_{8}$ no point of $E_{8}$ can belong to a rectangle of diameter $<\delta$ on which mean value of $f_{1}(x, y)$ is $\geqq M$. Thus the first of the sets (9) caunot have a positive measure. Similarly it can be proved that the second of them is also of measure zero, and in this way the proof of the theorem is completed.

Note on the differentiability of multiple integrals.

By

B. J es s en (Copenhagen),

J. Marcinkiewicz and A. Zygmund (Wilno).

$\S 1$.

Let $f\left(x_{1}, x_{2}, \ldots, x_{k}\right)=f(P)$ be an $L$-integrable function defined in the cell

$$
0 \leqslant x_{i} \leqslant 1
$$$$
(1 \leqslant i \leqslant k) \text {. }
$$

We shall say that the integral of the function $f$ is strongly differentiable at the point $P_{0}$, if

$$
\lim _{\delta(I) \rightarrow 0} \frac{1}{|I|} \int_{I} f(P) d P
$$

exists and is finite; here $I$ denotes any cell with sides parallel to the axes, contained in $S$, and containing $P_{0} ;|I|$ denotes the measure, and $\delta(I)$ the diameter of 1 . The limit (1) will be called the strong derivative of the integral of $f$ at the point $P_{0}$.

The following results have recently been established ${ }^{1}$ ).

Theorem A. There is a function $f(P) \in L$ such that its integral is nowhere strongly differentiable.

1) Theorem A was proved by S. Saks, Théorie de l'intégrale, Warszawa, 1933, pp. 1-288, esp. p. 232, Fund. Math. 22 (1934), 257-261, and independently by Busemann and Feller, Fund. Math. 22 (1934), 226-256. Theorem B, for bounded fanctions, was proved by Saks, Théorie de l'intégrale, p. 232, Busemann and Feller, loc. cit. F. Rie sz, Fund. Math. 22, p. 221-226, and, for functions of the elass $L^{p}, p>1$, by A. Z yg mund, Fund. Math, 23 (1934), 143-149 (see also Corrigenda at the end of this paper). 
Theorem B. If $f\left(P^{P}\right) \in L^{p}, p>1$, the strong derivative of the integral of $f(P)$ exists almost everywhere, and is equal to $f(P)$.

The object of this paper is to generalize and complete the above results, and to apply the generalizations to the theory of multiple Fourier series.

Given a function $f(P) \in L$, we write

$$
\begin{gathered}
f^{*}\left(P_{0}\right)=\sup _{I} \frac{1}{|I|} \int_{I}|f(P)| d P, \\
f_{*}\left(P_{0}\right)=\operatorname{Limsup}_{\delta(I) \rightarrow 0} \frac{1}{|I|} \int_{I}|f(P)| d P .
\end{gathered}
$$

We shall require the following lemmas due to Hardy and Littlewood ${ }^{1}$.

Lemma $A$. If the function $f(x), 0 \leqslant x \leqslant 1$, belongs to $I^{p}$, $p>1$, so does the function

$$
f^{*}(x)=\sup _{\xi_{1}<x<\xi_{a}} \frac{1}{\xi_{2}-\xi_{1}} \int_{\xi_{1}}^{\xi_{9}}|f(u)| d u
$$

and

$$
\int_{0}^{1}\left\{f^{*}(x)\right\}^{p} d x \leqslant C_{p} \int_{0}^{1}|f(x)|^{p} d x, \text { where } \quad C_{p}=2\left(\frac{p}{p-1}\right)^{p} .
$$

Lemma $B$. If $f(x) \log ^{+}|f(x)|, 0 \leqslant x \leqslant 1$, is integrable, so is $f^{*}(x)$, and

$$
\int_{0}^{1} f^{*}(x) d x \leqslant A \int_{0}^{1}|f| \log ^{+}|f| d x+B,
$$

where $A$ and $B$ are absolute constants.

$$
\S 2 .
$$

We shall-first consider the case $k=2$, and shall write $x, y$ for $x_{1}, x_{2}$. The letter $S$ will denote the square

$$
\text { (S) } \quad 0 \leqslant x \leqslant 1, \quad 0 \leqslant y \leqslant 1 \text {. }
$$

1) Acta Math., 54 (1930), 81-116; soo also Hardy, Littlowood and Poly a, Inequalities, Cambridge, 1934, p. 291
From Lemma A we deduce the following

Theorem 1. If $f(P) \in L^{p}, p>1$, then $f^{*}(P) \in L^{p}$, and

$$
\int_{s}\left\{f^{*}(P)\right\}^{p} d P \leqslant A_{p} \int|f(P)|^{p} d P
$$

with $A_{p}=C_{p}^{2}$, where $C_{p}$ is the constant of Lemma $A$.

We put

$$
\begin{aligned}
& g(x, y)=\operatorname{Sup}_{\eta_{1}<y<\eta_{2}} \frac{1}{\eta_{2}-\eta_{1}} \int_{\eta_{1}}^{\eta_{2}}|f(x, v)| d v \\
& h(x, y)=\operatorname{Sup}_{\xi_{1}<x<\xi_{2}} \frac{1}{\xi_{2}-\xi_{1}} \int_{\xi_{1}}^{\xi_{2}} g(u, y) d u .
\end{aligned}
$$

It will be shown in a moment that $g(x, y)$ is integrable, so that $h(x, y)$ is finite at almost every point of $S$. Using Lemma A, we may write

$$
\begin{gathered}
\int_{s} g^{p}(P) d P=\int_{0}^{1} d x \int_{0}^{1} g^{p}(x, y) d y \leqslant \\
\leqslant \int_{0}^{1} d x \cdot C_{p} \int_{0}^{1}|f(x, y)|^{p} d y=C_{p} \int_{S}|f(P)|^{p} d P .
\end{gathered}
$$

Hence $g \in L^{p}$, and therefore

(8) $\quad \int_{S} h^{p}(P) d P \leqslant C_{p} \int_{S} g^{p}(P) d P \leqslant C_{p}^{2} \int_{S}|f(P)|^{p} d P$.

Observing that, if $\xi_{1}<x<\xi_{2}, \eta_{1}<y<\eta_{2}$, then

$$
\begin{gathered}
\frac{1}{\left(\xi_{2}-\xi_{1}\right)\left(\eta_{2}-\eta_{1}\right)} \int_{\xi_{1}}^{\xi_{2}} \int_{\eta_{1}}^{\eta_{1}}|f(u, v)| d u d v \leqslant \\
\leqslant \frac{1}{\xi_{2}-\xi_{1}} \int_{\xi_{1}}^{\xi_{\xi_{1}}} g(u, y) d u \leqslant h(x, y),
\end{gathered}
$$

we obtain that

$$
f^{*}(P) \leqslant h(P),
$$

which, together with (8), completes the proof of (5). 
Theorem B is an easy consequence of Theorem $1^{1}$ ).

The following theorem contains Theorem $B$ as a special case.

Theorem 2. If $f(P) \log ^{+}|f(P)|$ is integrable over the square $S$, then, at almost every point $P$, the integral of $f$ is strongly differentiable, and the derivative is equal to $f(P)$.

The proof will be based on the following

Lemma $C$. Under the hypothesis of Theorem 2, the function $f_{*}(P)$, defined by the equation (3), is integrable, and

$$
\int_{s} f_{*}(P) d P \leqslant A \int_{s}|f(P)| \log ^{+}|f(P)| d P+B
$$

where $A$ and $B$ are the constants of Lemma $B$.

For, if $g(P)$ is defined by the equation (6), then, by Lemma B,

$$
\int_{0}^{1} g(x, y) d y \leqslant A \int_{0}^{1}|f(x, y)| \log +|f(x, y)| d y+B .
$$

Integrating this inequality with respect to $x$, we obtain

$$
\int_{s} g(P) d P \leqslant A \int_{s}|f(P)| \log +|f(P)| d P+B .
$$

It follows that, for almost every value of $y, g(x, y)$ is integrable as a function of $x$. Since, at almost every point $(x, y)$,

$$
\operatorname{Limsup}_{\xi_{1}<x<\xi_{2}} \frac{1}{\xi_{2}-\xi_{1}} \int_{\xi_{1}}^{\xi_{2}} g(u, y) d u=g(x, y)
$$

the first inequality in (9) gives

$$
f_{*}(P) \leqslant g(P)
$$

which, in view of (12), gives (11).

3) See A. Zygmund, loc. cit.

Theorem 1 can also be established by the argument which had previously been used to prove Theorem B (see Zyg mund, loc, cit.). That argument is independent of Lemmas $A$ and $B$, and may even be applied to obtain those lemmas.
Passing to the proof of Theorem 2, we observe, that applying (11) to the function $\lambda f$, where $\lambda>0$ is a constant, we obtain

$$
\int_{s} f_{*}(P) d P \leqslant A \int_{s}|f(P)| \log ^{+}|\lambda f(P)| d P+\frac{B}{\lambda} .
$$

Given an $\varepsilon>0$, we take $\lambda$ so large that $B / \lambda<\frac{1}{2} \varepsilon$, and put

$$
f(P)=\varphi(P)+\psi(P)
$$

where $\varphi$ is a continuous function, and

$$
\begin{gathered}
\int_{S}|\psi(P)| d P<\varepsilon \\
A \int_{S}|\psi(P)| \log ^{+}|\lambda \psi(P)| d P+\frac{B}{\lambda}<\varepsilon
\end{gathered}
$$

Applying the inequality (13) to the function $\psi(P)$, we obtain from (15)

$$
\int_{s} \psi_{*}(P) d P<\varepsilon
$$

This, together with (14), shows that the set $E(\varepsilon)$ of points $P$ where either $|\psi(P)|>\sqrt{\varepsilon}$, or $\psi^{*}(P)>\sqrt{\varepsilon}$, is of measure $<2 \sqrt{\varepsilon}$. Since

$$
\begin{aligned}
\frac{1}{|I|} \int_{i} f(P) d P & -f\left(P_{0}\right)=\left\{\frac{1}{|I|} \int_{I} \varphi(P) d P-\varphi\left(P_{0}\right)\right\}+ \\
& +\left\{\frac{1}{|I|} \int_{I} \psi(P) d P-\psi\left(P_{0}\right)\right\}
\end{aligned}
$$

where $P_{0} \in I$, we see that, outside the set $E(\varepsilon)$,

$$
\operatorname{Limsup}_{\delta(n) \rightarrow 0}\left|\frac{1}{|I|} \int_{I} f(P) d P-f\left(P_{0}\right)\right| \leqslant \psi_{*}\left(P_{0}\right)+\left|\psi\left(P_{0}\right)\right| \leqslant 2 \sqrt{\varepsilon} .
$$

Since the number $\varepsilon$ may be as small as we please, and the measure of $E(\varepsilon)$ tends to 0 with $\varepsilon$, the theorem follows.

Theorem 3. Under the hypothesis of Theorem 2, the function $f^{*}(P)$ belongs to $L^{\alpha}$ for every $0<\alpha<1$. 
For the proof we need the following

Lemma D. If the function $f(x)$ of Lemma $A$ is integrable, the function $f^{*}(x)$, defined by (4), belongs to every $L^{\alpha}, 0<\alpha<1$, and satisfies the inequality

$$
\left(\int_{0}^{1}\left\{f^{*}(x)\right\}^{\alpha} d x\right)^{1 / \alpha} \leqslant A_{a} \int_{0}^{1}|f(x)| d x, \quad(0<\alpha<1)
$$

where $A_{\alpha}$ depends on a only ${ }^{1}$.

From Lemmas $B$ and $D$, and using the functions $g, h$, defined by the equations $(6),(7)$, we obtain

(16) $\int_{0}^{1}\left\{\int_{0}^{1} h^{\alpha}(x, y) d x\right\}^{1 / \alpha} d y \leqslant A_{\alpha}\left(A \int_{S}|f(P)| \log ^{+}|f(P)| d P+B\right)$.

Now, in order to complete the proof of the theorem, it is sufficient to observe that, by Hölder's inequality,

$$
\int_{s} h^{\alpha}(P) d P \leqslant\left[\int_{0}^{1}\left\{\int_{0}^{1} h^{\alpha}(x, y) d x\right\}^{1 / \alpha} d y\right]^{\alpha},
$$

and to apply (10) and (16).

$$
\S 3
$$

Now we shall consider the case of arbitrary $k$. The reader will have no difficulty in verifying that Theorem 1 (with $A_{p}=C_{p}^{k}$ ), and so also Theorem B, remains true in the general case. More interesting is the following result.

Theorem 4. If $|f|\left(\log ^{+}|f|\right)^{k-1}$ is integrable over $S$, the integral of $f(P)$ is strongly differentiable at almost every point of $S$ to the value $f(P)$.

The proof is wholly analogous to the proof of Theorem 2, provided we can establish the following

1) This lemma, although not stated explicitly by Hardy and Littlowood, loc. cit., is a simple consequence of their results; see e. g. A. Zygmund, Trigonometrical Series, Warszawa, 1985, pp. 1-381, esp. p. 245 .
Lemma E. Let $f(x)$ be a function defined over the interval $0 \leqslant x \leqslant 1$, and let $f^{*}(x)$ be given by (4). Then, if $|f| \cdot\left(\log ^{+}|f|\right)^{r}$, $\cdot r=1,2, \ldots$, is integrable over $(0,1)$, so is $f^{*} \cdot\left(\log ^{+} f^{*}\right)^{r-1}$, and

$$
\text { (17) } \int_{0}^{1} f^{*} \cdot\left(\log ^{+} f^{*}\right)^{r-1} d x \leqslant A_{r} \int_{0}^{1}|f| \cdot\left(\log ^{+}|f|\right)^{r} d x+B_{r}
$$

where $A_{r}$ and $B_{r}$ depend on $r$ only.

This result is true for every positive $r^{1}$ ), but the special case just enunciated is sufficient for our purposes. Let

$$
\varphi(x)=x\left(\log ^{+} x\right)^{r-1} .
$$

It is well-known that it is enough to prove the lemma in the case when the function $f(x)$ is non-negative and non-increasing ${ }^{2}$ ). Then, the left-hand side of (17) is equal to

$$
\int_{0}^{1} \varphi\left(\frac{1}{x} \int_{0}^{x} f(t) d t\right) d x \leqslant \int_{0}^{1} \frac{d x}{x} \int_{0}^{x} \varphi\{f(t)\} d t
$$

by Jensen's inequality. Since $\varphi\{f(t)\}$ is a non-increasing function of $t$, the right-hand side of (18) does not exceed, in view of Lemma B,

$$
\begin{gathered}
A \int_{0}^{1} \varphi\{f\} \log ^{+} \varphi\{f\} d x+B= \\
=A \int_{0}^{1} f \cdot\left(\log ^{+} f\right)^{r-1} \log ^{+}\left\{f \cdot\left(\log ^{+} f\right)^{r-1}\right\} d x+B \leqslant A r \int_{0}^{1} f \cdot\left(\log ^{+} f\right)^{r} d x+B,
\end{gathered}
$$

and the lemma is established.

The proof of the following theorem may be left to the reader.

Theorem 5. Under the hypothesis of Theorem 4, the function $f^{*}(P)$ belongs to $L^{\alpha}$ for every $0<\alpha<1$.

If $f \cdot\left(\log ^{+}|f|\right)^{k}$ is integrable, so is the function $f^{*}$.

1) If $0<r<1$, we must replace $\log +f^{*}$ by $\log \left(2+f^{*}\right)$.

2) Hardy and Littlewood, loc. cit. Hardy, Littlewood and P6lya, Inequalities, p. 291. 
$\S 4$.

Let $f(P)$ be an integrable function defined in the $k$-dimensional cell $S$. The fundamental theorem of the Lebesgue theory of integration asserts that the integral of $f$ is differentiable, in the ordinary sense, at almost every point $P_{0}$, and the value of the derivative is equal to $f\left(P_{0}\right)$. By ordinary differentiability we mean the existence of the limit (1), where, however, the ratios of any two sides of the cell $I$ containing $P_{0}$ do not exceed a finite number, which may vary from point to point.

The following result completes Theorem 4 as well as Theorem A.

Theorem 6. Let $\alpha_{1}(t), \alpha_{2}(t), \ldots, \alpha_{k}(t)$ be arbitrary non-decreasing functions defined to the right of $t=0$, vanishing and contimuous for $t=0$, and positive for $t>0$. If the cells $I$ containing the point $P_{0}$ are of the form

$$
\xi_{i}^{\prime} \leqslant x_{l} \leqslant \xi_{i}^{\prime \prime}, \quad \xi_{i}^{\prime \prime}-\xi_{l}^{\prime}=\alpha_{i}(t), \quad i=1,2, \ldots, k,
$$

then the limit (1) exists and is equal to $f\left(P_{0}\right)$ at almost every point $P_{0}$.

So far as we are aware, this theorem has never been stated explicitly, although its proof is similar to that of the Lebesgue theorem mentioned at the beginning of this paragraph. This is not surprising, since Theorem 6 becomes interesting only in connexion with Theorem A, which result was obtained only very recently.

We shall not give the proof of Theorem 6 here, for this would be a mere repetition of the usual proof of the Lobergue theorem. It is sufficient to observe that the Vitali covering lemma, which plays the most fundamental part in the argument, remains valid for cells $I$ of the form just considered, and the proof is similar ${ }^{1}$ ). We leave it to the reader to fill in the details of the proof.

1) Seo e. g. the proof in Carath od ory's "Reelle Funltionen", p. 299 sqq. The sequence $W^{\prime}\left(P_{1}\right), W^{\prime}\left(P_{2}\right), \ldots$ of that proof (1. c., p. 301) may now bo arranged in the descending order of magnitude of the sides parallel to the $x_{1}$-axis.

Once Theorem 6 has been established, it is not difficult to see that it holds even when the cells $I$ do not contain the point $P_{0}$, provided the ratio $\left|I^{\prime}\right| /|T|$ does not exceed a number $\alpha\left(P_{0}\right)$, where $I^{\prime}$ is the emallost cell with sides parellel to the axes, baving $P_{0}$ as contre, and containing $I$.

We add that Theorem 6 is a special case of a more general theorem conconing the differentiability of functions of bounded variation.
The following theorem is an intermediate result between Theorems 4 and 6 .

Theorem \% Let $f(P)$ be a function in the k-dimensional cell $S$, and let $\alpha_{1}(t), \alpha_{2}(t), \ldots, \alpha_{r}(t)(2 \leqslant r \leqslant k)$, be $r$ functions having the properties enunciated in Theorem 6. Suppose that $f \cdot\left(\log ^{+}|f|\right)^{k-r}$ is integrable over $S$. Then, at almost every point $P_{0}$, the limit (1) exists and is equal to $f\left(P_{0}\right)$, provided the cells $I$ contain the point $P_{0}$ and are of the form

$$
\begin{array}{cc}
\xi_{i}^{\prime} \leqslant x_{i} \leqslant \xi_{i}^{\prime \prime} & (i=1,2, \ldots, k) \\
\xi_{j}^{\prime \prime}-\xi_{j}^{\prime}=\alpha_{j}(t) & (j=1,2, \ldots, r) .
\end{array}
$$

Let us suppose, for example, that $k=3, r=2$, and write $x, y, z$ for $x_{1}, x_{2}, x_{8}$, and $\alpha(t), \beta(t)$ for $\alpha_{1}(t), \alpha_{2}(t)$. Let $f_{*}\left(x_{0}, y_{0}, z_{0}\right)=$ $=f_{*}\left(P_{0}\right)$ denote the expression (3), where $I$ is of the form (20) $(k=3, r=2)$. It is sufficient to show that

$$
\int_{S} f_{*}(P) d P \leqslant A \int_{s}|f(P)| \log ^{+}|f(P)| d P+B
$$

with $A$ and $B$ independent of $f$, for then Theorem 7 may be obtained by an argument similar to that used in the proof of Theorem 4 .

Let

Since

$$
g(x, y, z)=\sup _{\zeta^{\prime}<z<\zeta^{\prime \prime}} \frac{1}{\zeta^{\prime \prime}-\zeta^{\prime}} \int_{\zeta^{\prime}}^{\zeta^{\prime \prime}}|f(x, y, w)| d w .
$$

$$
\int_{0}^{1} g(x, y, z) d z \leqslant A \int_{0}^{1}|f(x, y, z)| \log ^{+}|f(x, y, z)| d z+B
$$

we see that the function $g$ is integrable over $S$, and

$$
\int_{s} g(P) d P \leqslant A \int_{s}|f(P)| \log ^{+}|f(P)| d P+B
$$

A moment's consideration shows that

(23)

$$
f_{*}(x, y, z) \leqslant g_{*}(x, y, z)
$$

where

$$
g_{*}(x, y, z)=\lim _{\substack{\xi^{\prime \prime}<x<\xi^{\prime \prime} \\
\eta^{\prime}<<<\eta^{\prime \prime}}} \frac{1}{\left(\xi^{\prime \prime}-\xi^{\prime}\right)\left(\eta^{\prime \prime}-\eta^{\prime}\right)} \int_{\xi^{\prime}}^{\xi^{\prime \prime}} \int_{\eta^{\prime}}^{\eta^{\prime \prime}} g(u, v, z) d u d v\left\{\begin{array}{l}
\xi^{\prime \prime}-\xi^{\prime}=\alpha(t) \\
\eta^{\prime \prime}-\eta^{\prime}=\beta(t) .
\end{array}\right.
$$


By Theorem 6, we have $g_{*}(x, y, z)=g(x, y, z)$ at almost every point $(x, y, z)$, and this, together with the inequalities (23) and (22), gives $(21)$.

$$
\S 5 .
$$

We shall now prove that Theorem 4 cannot be strengthened. Let $\varphi(t), 0 \leqslant t<\infty$, be an increasing function satisfying the conditions

$$
\varphi(0)=0, \quad \operatorname{Liminf}_{t \rightarrow \infty} \frac{\varphi(t)}{t}>0,
$$

and let $L_{\varphi}$ denote the class of functions $f$ such that $\varphi(|f|)$ is integrable over $S$.

Theorem 8. If for every $f$ of $L_{\varphi}$ the integral of $f$ is strongly differentiable almost everywhere, then $\varphi(t)>c t\left(\log ^{+} t\right)^{k-1}$ for some constant $c>0$. In other words, $f \cdot\left(\log ^{+}|f|\right)^{k-1}$ is integrable over $\left.S^{1}\right)$.

We shall only consider the case $k=2$, the proof in the general case being essentially the same.

Lemma $\boldsymbol{F}$. Let $E$ be an arbitrary bounded and measurable set, and let $\sigma_{\alpha}(E), 0<\alpha<1$, denote the sum of all the rectangles $I$ for which

$$
|E I|>\alpha|I|
$$

Then, if the differentiability theorem is true for all functions of the class $L_{\varphi}$, the inequality

$$
\left|\sigma_{\alpha}(E)\right| \leqslant C \varphi\left(\frac{1}{\alpha}\right)|E|
$$

is true for all $E$ and all $\alpha$, the constant $C$ being independent of $\alpha$ and $E$.

The proof is indirect. We suppose that (26) is false, and prove, on this assumption, the existence of a function $f$ of $L_{p}$, for which the differentiation theorem is false.

1) This result is due to $\mathrm{Saks}$ and to Busemann and Feller. Saks has shown that, for every function $\varphi$ satisfying the hypothesis of Theorem 8 , there is an integrable function $f(x, y) \in L_{p}$ such that $\limsup _{h, k \rightarrow+0} \frac{1}{4 h k} \int_{x-h}^{x+h} \int_{y-k}^{y+k} f(u, v) d u d v=+\infty$ at every point $(x, y)$ (cf, this volume of Fundament(a, p. 285 sq(i). The argument of the text is dae to $B u s e m a n n$ and Follex.
Let the numbers $C_{n}>0$ be chosen in such a manner that

$$
\sum_{n} 1 / C_{n}<\frac{1}{2} \varphi(1)
$$

By our assumption, there exists, for every $n$, a bounded and measurable set $E_{n}$, and a number $\alpha_{n}, 0<\alpha_{n}<1$, such that

$$
\left|\sigma_{\alpha_{n}}\left(E_{n}\right)\right|>C_{n} \varphi\left(\frac{1}{\alpha_{n}}\right)\left|E_{n}\right| .
$$

We write $\sigma_{a_{n}}\left(E_{n}\right)=H_{n}$, and choose, for every $n$, a sequence of sets $H_{n}^{k}$ of diameter $<1 / n$, which are homothetic to $H_{n}$, cover $S$ except for a null set, and satisfy the condition

$$
\left.\sum_{k}\left|H_{n}^{k}\right|<2|S|=2^{1}\right)
$$

Let $E_{n}^{k}$ be the set derived by the same homothetical application by which $H_{n}$ is carried over in $H_{n}^{k}$. From

$$
\left|H_{n}\right|>C_{n} \varphi\left(\frac{1}{\alpha_{n}}\right)\left|E_{n}\right|
$$

it follows that

$$
\left|H_{n}^{k}\right|>C_{n} \varphi\left(\frac{1}{\alpha_{n}}\right)\left|E_{n}^{k}\right|
$$

1) That this is possible was shown by Busemann and Feller, loc. cit. p. 232. A somewhat simpler proof is the following.

We fix $n$, and write $H$ for $H_{n}$. Let $K$ be a closed subset of $H$, such that $|H|<2|K|$, and let $I$ be a square containing $K$. Let $S$ be divided into a finite number of equares $I^{p_{1}}$ of diameter $<1 / n$, and consider, for each $p_{1}$, the sets $H^{p_{1}}$ and $K^{p_{1}}$ derived from $H$ and $K$ by the same homothetical application by which $I$ is carried over in $I^{p_{1}}$. Writing $\varkappa=|K| /|I|$, and $S_{1}=S-\sum K_{n}^{p_{1}}$, we have clearly $\left|S_{1}\right|=1-x$. We now divide $S_{1}$ (except for a nall-set) into a finite or enumerable number of squares and proceed with each of these squares in exactly the same manner as we proceeded with $S$. Wo arrive then at two systems of sets $H^{p_{3}}$ and $K^{p_{2}}$, so that, if we write $S_{2}=S_{1}-\Sigma K^{p_{1}}$, we have $\left|S_{2}\right|=$ $=(1-x)\left|S_{1}\right|=(1-x)^{2}$. Continaing this process, and denoting by $H^{k}$ and $K^{k}$ the sets $H^{p_{1}}, H^{p_{3}}, \ldots$ and $K^{p_{1}}, K^{p_{3}}, \ldots$ respectively, the sets $H^{k}$ will satisfy the cunditions, since already the sets $K_{n}^{k}$ will cover $S$ except for a null-set, $\underset{k}{ }\left|K^{k}\right|=1$, and $\left|H^{k}\right|<2\left|K^{k}\right|$ for each $k$ 
Now put

in the set

$$
f_{n}(P)=1 / \alpha_{n}
$$

and

$$
S \sum_{k} E_{n}^{k}
$$

$$
f_{n}(P)=0
$$

at the remaining points of $S$, and let

Then

$$
f(P)=\operatorname{Sup} f_{n}(P)
$$

$$
\begin{aligned}
\int_{s} \varphi\{f(P)\} d P & \leqslant \sum_{n} \int_{S} \varphi\left\{f_{n}(P)\right\} d P \leqslant \\
& \leqslant \sum_{n} \sum_{k} \varphi\left(\frac{1}{\alpha_{n}}\right)\left|E_{n}^{k}\right| \leqslant \\
& \leqslant \sum_{n} \sum_{k} \varphi\left(\frac{1}{\alpha_{n}}\right) \frac{\left|H_{n}^{k}\right|}{C_{n} \varphi\left(\frac{1}{\alpha_{n}}\right)} \leqslant \\
& \leqslant 2|S| \sum_{n} \frac{1}{C_{n}}<\varphi(1),
\end{aligned}
$$

and so $f$ belongs to $L_{\varphi}$. On the other hand, for every $n$, almost every point $P$ of $S$ lies in at least one of the sets $H_{n}^{k}$, and hence (by the definition of $\sigma_{\alpha_{n}}\left(E_{n}\right)=H_{n}$ ) in a rectangle $I_{n}$ such that $\left|I_{n} E_{n}^{k}\right|>\alpha_{n}\left|I_{n}\right|$; also the diameter of $I_{n}$ tends to 0 as $n \rightarrow \infty$, since $I_{n}$ is contained in $H_{n}^{k}$, and the diameter of $H_{n}^{k}$ is $<1 / n$. Since $f(P) \geqslant 1 / \alpha_{n}$ for $P$ belonging to $S E_{n}^{k}$, we find

$$
\frac{1}{\left|I_{n}\right|} \int_{I_{n}} f(P) d P \geqslant \frac{1}{\left|I_{n}\right|} \cdot \frac{1}{\alpha_{n}}\left|I_{n} E_{n}^{k}\right| \geqslant \frac{1}{\left|I_{n}\right|} \cdot \frac{1}{\alpha_{n}} \cdot \alpha_{n}\left|I_{n}\right|=1 .
$$

Supposing that the differentiability theorem holds for $f$, we obtain $f(P) \geqslant 1$ for almost every point, and so

$$
\int_{s} \varphi\{f(P)\} d P \geqslant \varphi(1)
$$

which is in contradiction with (28), and so proves the lemma.

The proof of Theorem 8 in the case $k=2$ is now immediate.
If for $E$ we take the square $(S) 0 \leqslant x \leqslant 1,0 \leqslant y \leqslant 1$, then $\sigma_{\alpha}(E)$ contains the subset

Hence

$$
1 \leqslant x \leqslant 1 / \alpha, \quad 0 \leqslant x y \leqslant 1 / \alpha
$$

$$
\sigma_{\alpha}(E)>\int_{1}^{1 / \alpha} \frac{d x}{\alpha x}=\frac{1}{\alpha} \log \frac{1}{\alpha} \cdot|E|
$$

and so

$$
\frac{1}{\alpha} \log \frac{1}{\alpha}<C \varphi\left(\frac{1}{\alpha}\right)
$$

for all $\alpha$. Thence $\varphi(t)>c t \log ^{+} t$, and this is the desired result.

From Theorem 8 we obtain the following

Corollary. Let $\varepsilon(t), 0 \leqslant t<\infty$, be any bounded positive function tending to 0 as $t \rightarrow \infty$. Then there is a function $f(P)$ such that $f \cdot\left(\log ^{+}|f|\right)^{k-1} \in(|f|)$ is integrable, and yet the differentiation theorem does not hold for $f$.

Considering, for simplicity, the case $k=2$, we obtain from Theorem 2 and Lemma $\mathrm{F}$ the following elementary proposition.

Theorem 9. For any $0<\alpha<1$ there is a constant $C(\alpha)$ such that, with the notation of Lemma $F$,

$$
\left|\sigma_{\alpha}(E)\right| \leqslant C(\alpha)|E|
$$

for any bounded and measurable set $E$. If $C(\alpha)$ is chosen as small as possible, we have.

$$
0<\operatorname{Lim}_{\alpha \rightarrow 0} \inf \frac{C(\alpha)}{\frac{1}{\alpha} \log \frac{1}{\alpha}} \leqslant \operatorname{Lim}_{\alpha \rightarrow 0} \sup \frac{C(\alpha)}{\frac{1}{\alpha} \log \frac{1}{\alpha}}<\infty,
$$

so that $\frac{1}{\alpha} \log \frac{1}{\alpha}$ is the exact order of $C(\alpha)$ as $\alpha \rightarrow 0$.

The inequality (29), without the order result, was proved by Busemann and Feller (l. c.) and F. Riesz (l.c.), who on it based the proof of the differentiation theorem for bounded functions. It is, perhaps, worth while to mention that (29), without the exact order of $C(\alpha)$, is an immediate consequence of Theorem 1. In fact; since instead of a given set $E$ we may consider homothetic sets, let 
us assume that $E$ and $\sigma_{\alpha}(E)$ are contained in the square $(S) 0 \leqslant x \leqslant 1$, $0 \leqslant y \leqslant 1$. Let $f(P)=1$ in $E$, and $f(P)=0$ in $S-E$. Then the set $\sigma_{a}(E)$ is precisely the set of points where $f^{*}(P)>\alpha$. Hence, from (5)

$$
\left|\sigma_{\alpha}(E)\right| \leqslant A_{p} \alpha^{-p}|E|
$$

\section{$\S 6$.}

The methods which we used to prove the differentiability of multiple integrals, can also be applied to the problem of summability of multiple Fourier series. For simplicity we restrict ourselves to the case of double Fouriex series.

Let $K_{n}(x) \geqslant 0$ denote the Fejér kernel. If $f(x, y)$ is a function of period $2 \pi$ wilh respect to each of the variables $x, y$, the Fejerr means of the Fourier series of $f(x, y)$ are

(30) $\sigma_{m, n}(x, y)=\sigma_{m, n}(x, y ; f)=\frac{1}{\pi^{2}} \int_{-\pi}^{\pi} \int_{-\pi}^{\pi} f(u, v) K_{m}(x-u) K_{n}(y-v) d u d v$.

Theorem 10. If $f \log ^{\dagger}|f|$ is integrable, then $\sigma_{m, n}(x, y)$ tends to $f(x, y)$ at almost every point as $m, n \rightarrow \infty$.

Theorem 11. For every increasing function $\varphi(t), 0 \leqslant t<\infty$, satysfying the conditions

$$
\varphi(0)=0, \quad \operatorname{Liminf}_{t \rightarrow \infty} \frac{\varphi(t)}{t \log t}=0
$$
there is a function $f(x, y)$ belonging to $L_{\varphi}$, and such that $\sigma_{m, n}(x, y)$
does not converge almost everywhere $\left.{ }^{1}\right)$.

Theorem 10 could be deduced from Theorem 2 , but it is much simpler to base it on the following

Lemma G. Let $h(x), 0 \leqslant x \leqslant 2 \pi$, be a function such that $h \log ^{+}|h|$ is integrable. If $\tau_{m}(x)=\tau_{m}(x ; h)$ denote the Fejer sums of the Fourier series of $h(x)$, and $\tau^{*}(x)=\sup \left|\tau_{m}(x)\right|$, then

$$
\int_{0}^{2 \pi} \tau^{*}(x) d x \leqslant A^{\prime} \int_{0}^{2 \pi}|h| \log +|h| d x+B^{\prime \prime}
$$

where $A^{\prime}$ and $B^{\prime}$ are absolute constants $\left.{ }^{2}\right)$.

1) Thence it is not difficult to deduce the existence of a function $f(x, y)$ such that $\sigma_{m, n}(x, y)$ diverges almost everywhere.

2) Hardy and Littlewood, loc. cit.
In the case of $f(x, y)$ everywhere continuous, the expression $\sigma_{m, n}(x, y ; f)$ tends to $f(x, y)$ as $\left.m, n \rightarrow \infty{ }^{1}\right)$. Thence, arguing as in the proof of Theorem 2, we see that, in order to establish Theorem 10, it is sufficient to show that

(32) $\quad \int_{-\pi}^{\pi} \int_{-\pi}^{\pi} \sigma_{*}(x, y) d x d y \leqslant A \int_{-\pi}^{\pi} \int_{-\pi}^{\pi}|f| \log +|f| d x d y+B$

where $A$ and $B$ are absolute constants, and

$$
\sigma_{*}(x, y)=\operatorname{Limsup}_{m, n \rightarrow \infty}\left|\sigma_{m, n}(x, y)\right|
$$

Let, for fixed $y$,

$$
g(x, y)=\operatorname{Sup}_{m} \tau_{m}(x ;|f|) .
$$

Integrating this equation with respect to $x$, applying Lemma $G$, and then integrating with respect to $y$, we obtain

(33) $\int_{-\pi}^{\pi} \int_{-\pi}^{\pi} g(x, y) d x d y \leqslant 2 \pi A^{\prime} \int_{-\pi}^{\pi} \int_{-\pi}^{\pi}|f(x, y)| \log ^{+}|f(x, y)| d x d y+2 \pi B^{\prime}$

From (30) we have

$$
\begin{aligned}
\left|\sigma_{m, n}(x, y)\right| & \leqslant \frac{1}{\pi^{2}} \int_{-\pi}^{\pi} K_{n}(y-v) d v \int_{-\pi}^{\pi}|f(u, v)| K_{m}(x-u) d u \\
& \leqslant \frac{1}{\pi} \int_{-\pi}^{\pi} K_{n}(y-v) g(x, v) d v
\end{aligned}
$$

By the classical result of Lebesgue, the last expression tends to $g(x, y)$ at almost every point $(x, y)$; thence

$$
\sigma_{*}(x, y) \leqslant g(x, y) \quad \text { almost everywhere, }
$$

and (32) follows from (34) and (33).

The existence of $\lim \sigma_{m, n}(x, y)$ may be described as summability $(C, 1,1)$. Let $K_{m}^{\alpha}(x)$ be the $(C, \alpha)$ kernel. Replacing the product $K_{m}(x-u) K_{n}(y-v)$ in $(30)$ by $K_{m}^{\alpha}(x-u) K_{n}^{\beta}(y-v)$, we obtain expressions $\sigma_{m, n}^{\alpha, \beta}(x, y)$ which may be called the Cesàro sums of

1) See, e. g., Tonelli, Serie trigonometriche, p. 494. 
order $\alpha, \beta$ of the Fourier series of $f(x, y)$. Without essential changes, the argument given. above shows that almost everywhere

$$
\sigma_{m, n}^{\alpha, \beta}(x, y) \rightarrow f(x, y)
$$

as $m, n \rightarrow \infty$, provided that $\alpha>0, \beta>0$. We omit the details of the proof $\left.{ }^{1}\right)$. Similarly we can prove that, under the hypothesis of Theorem 10, the Fourier series of $f(x, y)$ is summable almost everywhere by Abel's method ${ }^{2}$ ) to the value $f(x, y)$.

Passing to the proof of Theorem 11, we observe that, on account of Theorem 8 , there is a function $f(x, y)$ of $L_{\varphi}$, whose integral is not differentiable (in the strong sense) at every point of a set $E$ of positive measure. This function is non negative, and so at almost every point of $E$ we have

$$
\begin{aligned}
& \text { (35) } f(x, y) \leqslant \liminf _{h_{1}, h^{\prime}, k^{\prime} \rightarrow+0} \frac{1}{\left(h+h^{\prime}\right)\left(k+k^{\prime}\right)} \int_{x \rightarrow h}^{x+h^{\prime}} \int_{y-k}^{y+k^{\prime}} f(u, v) d u d v< \\
& <\operatorname{Limsup}_{h, h^{\prime}, k^{\prime} \rightarrow+0} \frac{1}{\left(h+h^{\prime}\right)\left(k+k^{\prime}\right)} \int_{x \rightarrow h}^{x+h^{\prime} y+k^{\prime}} \int_{y-k} f(u, v) d u d v .
\end{aligned}
$$

The first inequality in (35) follows from the fact that, for every $\varepsilon>0$, we can find a bounded function $g(x, y)$ satisfying the inequalities

$$
f(x, y)-\varepsilon<g(x, y)<f(x, y)
$$

except in a set of measure $<\varepsilon$, and from the fact that the differentiation theorem holds for bounded functions." We may of course suppose that the second inequality of (36) is satisfied every where ${ }^{3}$ ). Taking $\varepsilon$ small enough, and substracting $g$ from $f$, we obtain a positive

1) The argument is similar to that of the text, if we use a lemma analogous to Lemma G, the kernel $K_{m}(x)$ being replaced by $\left|K_{m}^{\alpha}(x)\right|$. The fact that $K_{m}^{\alpha}(x)$ may assume negative values when $0<\alpha<1$, is not essential for the proof.

2) A double series $\Sigma \Sigma a_{m n}$ is said to be summable by Abel's method of summation if

$$
\lim \Sigma \Sigma a_{m n},^{m} \varrho^{n}
$$

exists for $r \rightarrow 1, \varrho \rightarrow 1$.

3) In view of the Lebergue theorem mentioned at the boginning of $\& 4$, the first inequality in (35) is, in fact, an equality. function, which we shall again denote by $f$, such that in a set $E_{1}$ of positive measure

(37) $\lim _{h, k, h^{\prime}, k^{\prime} \rightarrow+0} \frac{1}{\left(h+h^{\prime}\right)\left(k+k^{\prime}\right)} \int_{x \rightarrow h}^{x+h^{\prime}} \int_{y=-k}^{y+k^{\prime}} f(u, v) d u d v>4 \mu f(x, y)$,

where $\mu>0$ is an arbitrary, but fixed, constant. From (37) we see that, for $(x, y)$ belonging to $E_{1}$, we have

(38) $\quad \limsup _{h, k \rightarrow+0} \frac{1}{4 h k} \int_{x \rightarrow-k}^{x+h} \int_{y=k}^{y+k} f(u, v) d u d v>\mu f(x, y)$

Taking into account that $K_{m}(x)$ is non-negative, that (39)

$$
K_{m}(x)>C m \text { for }|x|<\frac{1}{m}
$$

where $C>0$ is an absolute constant, and putting $m=[1 / h], n=[1 / k]$, where the numbers $h, k$ are those of the inequality (38), we obtain from (30) and (39) that

$$
\text { (40) } \begin{aligned}
\sigma_{m, n}(x, y) \geqslant & \frac{1}{\pi^{2}} \int_{x-h}^{x+h} \int_{y+k}^{y+k} f(u, v) K_{m}(u-x) K_{n}(v-y) d u d v \geqslant \\
& \geqslant C_{1} \frac{1}{4 h k} \int_{x-h}^{x+h} \int_{y-k}^{y+k} f(u, v) d u d v,
\end{aligned}
$$

$C_{\imath}$ denoting a positive absolute constant. Supposing that the number $\mu$ of (38) exceeds $1 / C_{1}$, we deduce from (38) and (40) that

$$
\operatorname{Limsup}_{m, n \rightarrow \infty} \sigma_{m, n}(x, y)>f(x, y)
$$

at almost every point of $E_{1}$. This shows that, at almost every point of $E_{1}, \sigma_{m, n}(x, y)$ does not tead to $f(x, y)$. Since, in view of $(30)$, $\int_{-\pi}^{\pi} \int_{-\pi}\left|\sigma_{m, n}(x, y ; f)-f\right| d x d y \leqslant \sigma_{m, n}(0,0 ; F) \rightarrow 0, \quad(m, n \rightarrow \infty)$
where $F(u, v)=I^{\pi} f^{\pi}|f(x+u, y+v)-f(x, y)| d x d y$ is a continuous where $F(u, v)=\int_{-\pi}^{\pi} \int_{-\pi}^{\pi}|f(x+u, y+v)-f(x, y)| d x d y$ is a continuous
function vanishing at $u=0, v=0$, the sequence $\sigma_{m, n}$ tends in mean function vanishing at $u=0, v=0$, the sequence $\sigma_{m, n}$ tends in mean
to $f$. From this and (41) we deduce that the sequence $\sigma_{m, n}$ diverges almost everywhere in $E_{1}$, and Theorem 11 follows ${ }^{1}$ ).

1) It is easy to see that, for the Saks function $f(x, y)$ mentioned in footnote ${ }^{1}$ ) on p. 226, we have $\operatorname{Limsup}_{m, n} \sigma_{m, n}(x, y)=+\infty$ at every point $(x, y)$. 
We add that the function

$$
\sigma^{*}(x, y)=\operatorname{Sup}_{m, n}\left|\sigma_{m, n}(x, y)\right|
$$

satisfies theorems analogous to Theorems 1,2 and 5 . The same may be said of the Abel and $(C, \alpha, \beta)(\alpha>0, \beta>0)$ means.

Corrigenda to the paper "On the differentiability of multiple integrals" by A. Z y g mund (Fundamenta Mathematicae, vol. 23, p. 143-149).

Prof. Banach kindly called my attention to the fact that the proof of the lemma on p. 145 is incomplete, for the argument on p. 146 , line 15 , is valid in the case $k=l$ only. The proof may be completed in various ways, and, in particular, as follows.

We have to show, that, given any fanctions $h=h(x, y), k=k(x, y)$, the expression.

$$
\mu(u, v)=\iint_{S} L_{h}(x-u) L_{k}(y-v) d x d y
$$

satisfies an inequality $I_{q}[\mu] \leqslant A_{q}$, whore $A_{q}$ depends on $q$ only. In the first place, we observe that, given any function $g=g(x)$, the function $\lambda(u)=\int_{0}^{1} L_{g}(u-x) d x$ belongs to every $L^{q}$, and the integral of $\lambda q(u)$ over $0 \leqslant u \leqslant 1$ does not exceed a constant $B_{q}^{q}$. This is an analogue, for the one-dimensional space, of the result which we have to prove; the proof follows by an argument similar to that of section 4 of the paper. Assuming this, let us consider any of the terms of the $\operatorname{sum} \underset{k, l=1}{\sum_{l=1}^{q}}\left\{I_{l=1}^{q}(k), \ldots \prod_{j=1}^{q}(l) \ldots\right\}$ on p. 146, line 9. Suppose first that $k$. $l=2$. Integrating first with respect to $x_{3}, \ldots, x_{q}, y_{3}, \ldots, y_{q}$, and then with respect to $x_{1}, x_{2}, y_{1}, y_{2}$, we obtain

$$
\int_{0}^{1} \int_{0}^{1} d x_{1} d y_{2} \mu^{q-2}\left(x_{1}, y_{2}\right) \int_{0}^{1} L_{2 h_{2}}\left(x_{1}-x_{2}\right) d x_{2} \int_{0}^{1} L_{2 k_{1}}\left(y_{1}-y_{1}\right) d y_{1} .
$$

Applying Hölder's inequality with the three exponents $q /(q-2), q$, $q$, we see that the integral does not exceed $I_{q}^{q-2}[\mu] B_{q}^{2}$. If $k=l$, e.g. $k=l=1$, the integra] is equal to $\int_{0}^{1} \int_{0}^{1} \mu^{q-1}\left(x_{1}, y_{1}\right) d x_{1} d y_{1} \leqslant I_{q}^{q-1}[\mu]$. Collecting the terms, we finally obtain

$$
I_{q}^{q}[\mu] \leqslant C_{q}\left\{I_{q}^{q-1}[\mu]+I_{q}^{q-2}[\mu]\right\},
$$

where $C_{q}$ depends on $q$ only. It is plainly sufficient to consider the case when $h(x, y)$ and $k(x, y)$ have a positive minimum. Then $I_{q}[\mu]$ is finite, and so does not exceed the largest root of the equation $t q-C_{q}\left(t q-1+t^{q-2}\right)=0$. This complotes the proof.

\section{On the strong derivatives of functions of intervals.}

By

\section{S. Saks (Warszawa).}

Introduction. Given a set of $2 p$ numbers $a_{1} \leqslant b_{1}, a_{2} \leqslant b_{2}, \ldots$, $a_{p} \leqslant b_{p}$ the set of points $\left(x_{1}, x_{2}, \ldots, x_{p}\right)$ such that $a_{i} \leqslant x_{i} \leqslant b_{i}$ for $i=1,2, \ldots, p$, will be denoted as the in terval $\left[a_{1}, b_{1} ; a_{2}, b_{2} ; \ldots ; a_{p}, b_{p}\right]$ of the $p$-dimensional space $\Re_{p}$. If $F(I)$ is an addditive function of intervals and $I_{0}$ an interval in $\Re_{p}$, then $\mathrm{V}\left(F ; I_{0}\right)$ will denote the total (absolute) variation of $F$ over $I_{0}$. If $F(I)$ is a function of intervals of bounded variation then it may be extended as a completely addditive function of sets to the family of all sets measurable $\left.{ }^{1}\right)(B)$; accordingly, in this case, $\nabla(F ; A)$ for any set $A$ measurable $(B)$ will mean the total variation of $F$ over $A$.

If $\left(x_{1}, x_{2}, \ldots, x_{p}\right)$ is a point in the space $\Re_{p}$ and $F(I)$ a function of intervals, then the lower and upper limits of the quotient $F(I) /$ meas $I$, where $I$ is an arbitrary interval containing $\left(x_{1}, x_{2}, \ldots, x_{p}\right)$ and $\mathrm{d}(I) \rightarrow 0$, will be called the lower and upper strong derivates of $F(I)$ at the point $\left(x_{1}, x_{2}, \ldots, x_{p}\right)$, and denoted by $F^{*}\left(x_{1}, x_{2}, \ldots, x_{p}\right)$ and $\bar{F}^{*}\left(x_{1}, x_{2}, \ldots, x_{p}\right)$ respectively. In the case when they are equal we shall write $F^{*}\left(x_{1}, x_{2}, \ldots, x_{p}\right)$ for their common value, that will be called the strong derivativea) of $F(I)$ at the point considered. $F^{\prime}\left(x_{1}, x_{2}, \ldots, x_{p}\right)$ will as usually denote the derivative of $F(I)$ in the ordinary sense. In the case

1) See for instance de la Vallée-P oussin, Intégrales de Lebesgue, Fonctions d'ensemble, Classes de Baire, 2。 éd., Paris (1934), pp. 88-95; Saks, Théorie de l'intégrale, Warszawa (1933), p. 250.

3) Some problems concerning the strong derivation of additive functions of intervals have been recently diseussed in a series of papers published in these Fundamenta; see the list at the ond of this note. 